\title{
Will cardiac surgeons even turn pumpkins into carriages?
}

\author{
Philippe Menasché, MD, PhD
}

\footnotetext{
From the Department of Cardiovascular Surgery, Université Paris Descartes, Sorbonne Paris Cité, INSERM U-970, Hôpital Européen Georges Pompidou 20, Paris, France.

Disclosures: Author has nothing to disclose with regard to commercial support.

Received for publication Nov 22, 2017; accepted for publication Dec 18, 2017; available ahead of print Jan 31, 2018.

Address for reprints: Philippe Menasché, MD, PhD, Department of Cardiovascular Surgery, Hôpital Européen Georges Pompidou 20, rue Leblanc, Paris 75015, France (E-mail: philippe.menasche@aphp.fr).

J Thorac Cardiovasc Surg 2018;155:1647-9

$0022-5223 / \$ 36.00$

Copyright (c) 2017 by The American Association for Thoracic Surgery

https://doi.org/10.1016/j.jtcvs.2017.12.067
}

The take-home message of the paper by Rosengart and colleagues ${ }^{1}$ is that it is timely to revisit the strategy of "regenerative" medicine by shifting from the transplantation of exogenous stem cells to the exploitation of the alleged ability of some cardiac cells, particularly the fibroblasts, to change their phenotype in response to appropriate signals. This paradigm shift is primarily based on the seminal work of the Nobel Prize winner Shinya Yamanaka, ${ }^{2}$ who has shown that skin fibroblasts, when exposed to a bunch of cell fate-changing transcription factors, can be converted back to pluripotent stem cells. They can then be redifferentiated into various cell types including cardiomyocytes, thereby demonstrating that adult somatic cells, once thought to be terminally differentiated, were actually malleable enough to cross their lineage boundaries and adopt a new phenotype through large-scale changes in gene expression and chromatin status. One step further, the work from Srivastava's group ${ }^{3}$ has unraveled a shortcut pathway allowing the direct conversion of fibroblasts into cardiomyocyte-like cells (without the intermediate step of the pluripotent stage), thereby confirming that cell fate was amenable to dynamic changes provided the target cells were exposed to appropriate triggers, in this case a cocktail of 3 growth factors. Although this study was initially conducted in murine fibroblasts, it has since then been successfully extended to their human counterparts. ${ }^{4}$

The major strength of the paper by Rosengart and colleagues ${ }^{1}$ is to build on these discoveries by raising the challenging hypothesis that it might be possible to transform the fibroblasts that populate postinfarction scars into newly functioning, beating cardiomyocytes. Needless to say, such an in situ conversion could dramatically improve the outcome of patients suffering from heart failure as a consequence of extensive areas of scarred myocardium and might overcome some of the major hurdles associated with conventional exogenous stem cell transplantation and that are highlighted in their paper. This enthusiasm, however, needs to be cautiously tempered by the recognition of some major, and yet still unsettled, issues.

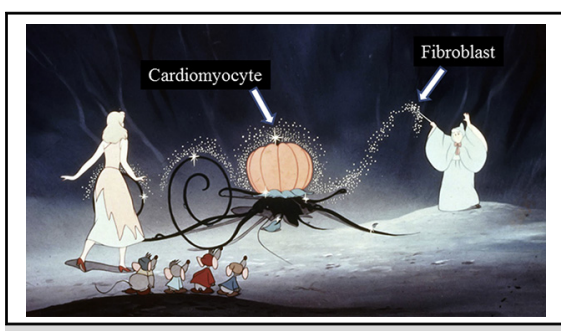

It takes more than a magic wand to turn fibroblasts into new regenerative cardiomyocytes.

\section{Central Message}

Until now, attempts at regenerating the infarcted myocardium have relied on the transplantation of exogenous cells. A more efficient strategy could consist of leveraging the phenotypic plasticity of endogenous in-scar fibroblasts through the delivery of reprogramming agents, allowing to turn them into new cardiomyocytes.

See Article page 1642 .

A first issue pertains to the efficiency of the procedure. In a mouse model of myocardial infarction, Song and colleagues, ${ }^{5}$ using an optimal combination of reprogramming factors, have reported that the functional fibroblastderived converted cardiomyocytes only comprised about $6 \%$ of those of the injured area. This low efficiency rate is due to several factors well identified during fibroblast or astroglial reprogramming into neurons, such as divergence from the targeted differentiation path into an alternative fate, silencing of reprogramming factors, ${ }^{6}$ or oxidative stress upon transdifferentiation. ${ }^{7}$ Even though the myocardial microenvironment seems more permissive to reprogramming than plastic tissue culture dishes ${ }^{8}$ and injury, by itself, may facilitate cellular reprogramming, as exemplified by the atrial-to-ventricular transdifferentiation that contributes to zebrafish cardiac ventricular regeneration, ${ }^{9}$ the efficiency of the procedure could, at the opposite, be complicated by the greater resistance of human cells to reprogramming as compared with their murine counterparts. Manipulations might thus be required to lower this threshold and make the cells more permissive to the reprogramming triggers, thereby adding an additional layer of technical and regulatory complexity.

It further remains to determine which triggers (lineagerestricted transcription factors involved in early embryonic cardiac development, exosomes, their noncoding nucleic acid cargo, small molecules regulating epigenetics or a 
combination thereof) and how much of them would be required to cause a cardiogenic conversion of myofibroblasts of sufficient magnitude to elicit a clinically relevant improvement in heart function. This dosing issue is far from being trivial, as shown by the finding that stoichiometry of the transcription factors influences the efficiency and quality of fibroblast to cardiomyocyte reprogramming. ${ }^{10}$ Importantly, the increase in postinfarction functional recovery achieved in rodents by these reprogramming strategies has to be cautiously interpreted in view of their limited translatability to the human setting, and the results of a large animal study are thus eagerly awaited to help clarifying this issue. It is also noteworthy that a robust regeneration in infarcted myocardium does not only require an increased provision of new cardiomyocytes but also their nutritive support by the restoration of an effective blood supply.

A second issue pertains to the safety of the procedure from the dual perspective of the reprogramming agents themselves and their consequences on the myocardium. So far, as we still do not know which of these triggers would be the most efficient, we equally ignore the potential hazards associated with the delivery of these compounds with regard to local unwanted genetic/epigenetic changes and/ or off-target ectopic gene expression. One can easily figure out the safety data that would be required by the regulatory authorities before approval of any of these agents for human use. This issue would be further complicated if, as alluded to in the manuscript, downregulation of p63 was required as an additional manipulation to facilitate reprogramming of human cells because p63 is a transcription factor that exhibits isoform-specific expression involved in either cell-cycle arrest and apoptosis or, conversely, cell proliferation. ${ }^{11}$ In contrast, assuming these interventions become truly efficient, another safety issue would be the regulation of the reprogramming events to avoid an uncontrolled proliferation of the converted cells, particularly if they first go through a transient amplifying progenitor state. Fail-safe mechanisms should thus have to be considered for appropriately regulating these events.

In addition, the authors rightly point out that one of the likely causes of failure of several cell therapy trials has been the inability of the grafted cells to appropriately couple with the host cardiomyocytes. This patterning issue might also be relevant to converted fibroblasts. Namely, it could be challenging to ensure that all the reprogrammed fibroblasts align with the host cells in such a homogeneous fashion that an efficient electromechanical integration can be achieved, as evidence is still lacking that this coupling, which has been successfully reported following the in vivo cardiomyocytic conversion of murine fibroblasts, ${ }^{12}$ is translatable to the human heart. If it was not the case, an only partial reprogramming could compromise the functional efficacy of the procedure and result in inhomogeneities of electrical conduction at the graft-host interface, setting the stage for worrisome arrhythmias.

A third issue pertains to the delivery of the reprogramming agents. Among the causes of failure of current stem cell trials, the authors also appropriately mention the low efficiency of most delivery techniques, regardless of the route which is used (intracoronary, endomyocardial, transepicardial). This issue also applies to the transfer of the agents intended to convert the in-scar fibroblasts. Among them, gene vectors combining a high transfection potential and a satisfactory safety profile could benefit from the clinical resurgence of gene therapy trials, particularly if they could be efficiently delivered into the pericardial cavity by a minimally invasive approach. ${ }^{13}$ Gene-associated issues could be overcome by locally delivered chemical inducers of cellular reprogramming but because it is unlikely that a single snapshot injection in the myocardium could efficiently reprogram a sufficient mass of fibroblasts, engineering technologies would need to be developed to allow for a time-controlled delivery. At the end, progress in nanotechnologies might allow systemically injected compounds bearing site-specific receptors to be driven toward the target areas for locally harnessing the endogenous cells' regenerative potential.

In conclusion, the multiple pitfalls of exogenous stem cell therapy certainly justify the further exploration of an alternate strategy based on a regeneration "from within" through leveraging the plasticity of cell fate for converting fibroblasts present in scar tissue into new contractile cardiomyocytes. The appropriate manipulation of gene networks driving cell fate decisions could thus represent a promising strategy for alleviating heart failure. However, although the paper by Rosengart and colleagues ${ }^{1}$ provides a severe conviction of conventional stem cell therapies, it is fair to acknowledge that this first wave of clinical trials has paved the way for what they propose. Namely, the reduction of postinfarction fibrosis has been commonly reported after experimental stem cell transplantation and attributed to paracrine signaling. The approach advocated by Rosengart

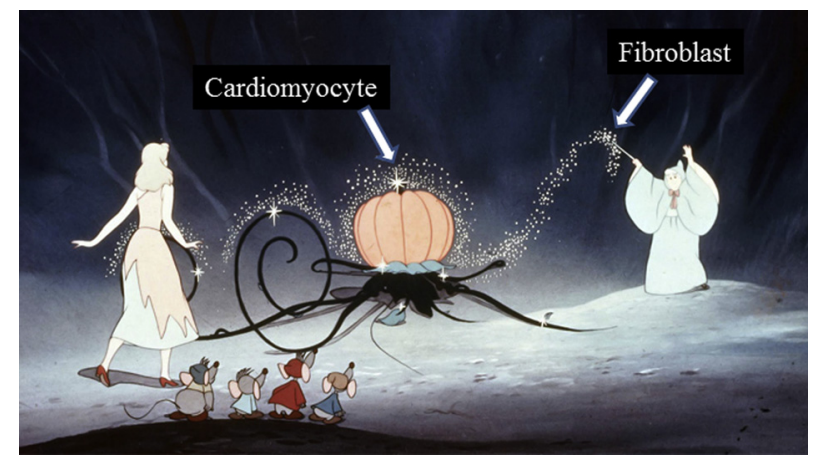

FIGURE 1. Although conversion of in-scar fibroblasts into cardiomyocytes is experimentally well documented, there is more to do than using a magic wand to turn this biological event into an efficacious therapy of heart failure. 
and colleagues ${ }^{1}$ tries nothing else than magnifying this event through the use of more specific fibroblast-targeted reprogramming agents. However, multiple practical issues still need to be addressed before we can safely leverage the adaptive cellular reprogramming events that occur in vivo in several tissues as part of the body's self-repair mechanisms and make them therapeutically relevant. Clearly, for the time being, the practicing cardiac surgeon still lags behind the fairy tale of Cinderella for turning the pumpkin into a carriage (Figure 1).

\section{References}

1. Rosengart TK, Patel V, Sellke FW. Cardiac stem cell trials and the new world of cellular reprogramming: time to move on. J Thorac Cardiovasc Surg. 2018;155: 1642-6.

2. Takahashi K, Yamanaka S. Induction of pluripotent stem cells from mouse embryonic and adult fibroblast cultures by defined factors. Cell. 2006;126:663-76.

3. Ieda M, Fu JD, Delgado-Olguin P, Vedantham V, Hayashi Y, Bruneau BG, et al. Direct reprogramming of fibroblasts into functional cardiomyocytes by defined factors. Cell. 2010;142:375-86.

4. Cao N, Huang Y, Zheng J, Spencer CI, Zhang Y, Fu J, et al. Conversion of human fibroblasts into functional cardiomyocytes by small molecules. Science. 2016; $352: 1216-20$
5. Song K, Nam YJ, Luo X, Qi X, Tan W, Huang GN, et al. Heart repair by reprogramming non-myocytes with cardiac transcription factors. Nature. 2012;485: 599-604.

6. Treutlein B, Lee QY, Camp JG, Mall M, Koh W, Shariati SA, et al. Dissecting direct reprogramming from fibroblast to neuron using single-cell RNA-seq. $\mathrm{Na}$ ture. 2016;534:391-5.

7. Gascon S, Muren E, Masserdotti G, Ortega F, Russo GL, Petrik D, et al. Identification and successful negotiation of a metabolic checkpoint in direct neuronal reprogramming. Cell Stem Cell. 2016;18:396-409.

8. Ebrahimi B. In vivo reprogramming for heart regeneration: a glance at efficiency, environmental impacts, challenges and future directions. J Mol Cell Cardiol. 2017;108:61-72.

9. Zhang R, Han P, Yang H, Ouyang K, Lee D, Lin YF, et al. In vivo cardiac reprogramming contributes to zebrafish heart regeneration. Nature. 2013;498 497-501.

10. Ma H, Wang L, Yin C, Liu J, Qian L. In vivo cardiac reprogramming using an optimal single polycistronic construct. Cardiovasc Res. 2015;108: 217-9.

11. Lin C, Li X, Zhang Y, Guo Y, Zhou J, Gao K, et al. The microRNA feedback regulation of p63 in cancer progression. Oncotarget. 2015;6:8434-53.

12. Qian L, Huang Y, Spencer CI, Foley A, Vedantham V, Liu L, et al. In vivo reprogramming of murine cardiac fibroblasts into induced cardiomyocytes. Nature. 2012;485:593-8.

13. Laakmann S, Fortmüller L, Piccini I, Grote-Wessels S, Schmitz W, Breves G, et al. Minimally invasive closed-chest ultrasound-guided substance delivery into the pericardial space in mice. Naunyn Schmiedebergs Arch Pharmacol. 2013;386:227-38. 